\title{
Feria vocacional y elección vocacional en estudiantes de quinto de secundaria
}

\section{RESUMEN}

José Mercedes Vidal Sevilla

jomevise@gmail.com

https://orcid.org/0000-0001-7005-1557

Universidad César Vallejo. Lima - Perú

Teobaldo Carlos Sevilla Muñoz

Carlossevillam67@gmail.com https://orcid.org/0000-0002-5468-9400 Universidad Cesar Vallejo. Lima - Perú

Eufemia María Martínez Rueda eufemiamr2@gmail.com https://orcid.org/0000-0001-5265-0740 Universidad César Vallejo. Lima - Perú

Rocío Andrea Nauca Guzmán rocioandrea8800@gmail.com https://orcid.org/0000- 0002-3497-3711 Universidad César Vallejo. Lima - Perú

Melquiades Cordova Ludeña melqiadescordova@gmail.com https://orcid.org/0000-0002-5223-5763 Universidad Cesar Vallejo. Lima - Perú

La elección de una carrera profesional en estudiantes que terminan la secundaria es un proceso complejo y es importante que cuenten con la orientación vocacional adecuada, por ello este artículo científico tiene como objetivo determinar cómo se relaciona la feria vocacional y la elección de una carrera en estudiantes de quinto de secundaria de la IE Tupac Amaru II SJL 2020. La investigación es no experimental, descriptiva y correlacional con enfoque cuantitativo. En la muestra participaron 60 estudiantes de quinto año de nivel secundaria. Se aplicaron dos encuestas para recoger la información. Los resultados arrojaron una correlación de 0.000 y una significancia bilateral de 0.999 > a 0.05 por lo que se concluyó que no existe una relación significativa entre feria vocacional y elección vocacional entre los estudiantes de quinto de la IE Tupac Amaru II SJL 2020.

Palabras clave: elección profesional, formación profesional, futuro, trabajo 


\title{
Vocational fair and vocational choice in fifth grade high school students
}

\begin{abstract}
The choice of a professional career in students who finish high school is a complex process and it is important that they have the appropriate vocational guidance, therefore this scientific article aims to determine how the vocational fair is related to the choice of a career in students fifth year of secondary school of the IE Tupac Amaru II SJL 2020. The research is non-experimental, descriptive and correlational with a quantitative approach. Sixty fifth-year high school students participated in the sample. Two surveys were applied to collect the information. The results yielded a correlation of 0.000 and a bilateral significance of $0.999>$ to 0.05 , so it was determined that there is no significant relationship between vocational fair and vocational choice among fifth-year students of IE Tupac Amaru II SJL 2020.
\end{abstract}

Keywords: career choice, vocational training, future, work

Artículo recibido: 20 diciembre. 2021 Aceptado para publicación: 10 enero 2022 Correspondencia: carlossevillam67@gmail.com Conflictos de Interés: Ninguna que declarar 


\section{INTRODUCCIÓN}

Los estudiantes de quinto año enfrentan un gran desafío al terminar la secundaria por la responsabilidad de tomar una decisión relevante para su persona, con la implicancia de la participación de familiares que resulta compleja, porque tiene que elegir una carrera universitaria o superior que va a impactar tanto en su desarrollo profesional y personal de su futuro. En este proceso las universidades son un soporte para ayudar a los estudiantes que están en la transición a la educación superior, mediante la organización de ferias vocacionales.

Según, la Oficina de Orientación de la Universidad de Costa Rica (2019), la Feria Vocacional es una estrategia para ayudar a los estudiantes que egresan de la secundaria y brindarles información sobre las opciones que ofrece la universidad en la elección de una carrera que permita desarrollar sus habilidades, competencias profesionales y mercado de trabajo. En esa línea, Semenkova (2016) refiere que las condiciones socioeconómicas contemporáneas han cambiado significativamente el enfoque de la educación profesional y la autodeterminación profesional de los jóvenes y el sistema educativo debe orientar a los jóvenes que están esperando respuestas a interrogantes como ¿Qué profesiones serán demandadas en el futuro?, ¿Las especialidades elegidas satisfacen las condiciones socioeconómicas en constante cambio?; preguntas que surgen por el aumento de la competencia en el mercado laboral y que obliga a la educación una reestructuración motivacional para resolver el problema de la orientación profesional de los jóvenes .Por otro lado, en la Expo-estudiante (2019) se buscó responder las expectativas de los padres y las necesidades e intereses laborales de los estudiantes como ¿Qué estudiar? ¿Dónde estudiar? ¿con que habilidades cuenta para estudiar una carrera? ¿Cómo está el mercado laboral?; Al respecto, Sánchez (2017) refiere que el empleo tiene un papel importante en la vida de los individuos y las actividades de orientación abarcan desde inicios del siglo $\mathrm{XX}$, como resultado de la problemática derivada de la industrialización, cuando las personas encontraron la necesidad de aprender algunos oficios.

De acuerdo a Thenmozhi (2018) es importante tener en cuenta que la elección vocacional debe decidirse más en función de la capacidad, el interés y la aptitud del individuo, más que en las aspiraciones de los padres únicamente. Según Pronabec (2019) en la Beca 18, se brinda orientación vocacional a los jóvenes entre 16 a 18 años, para que puedan elegir una carrera profesional; sugieren a los padres que sus hijos descubran la profesión que les 
apasiona de acuerdo a sus intereses profesionales, capacidad intelectual, formas de aprendizaje, estilos de estudio y personalidad.

Sobre el tema, Olivares y Quezada (2013) establecen que una feria vocacional es un punto de encuentro donde las instituciones educativas y/o empresas ofrecen información sobre las carreras profesionales, modalidades, duración, costo de ellas y bolsa de trabajo. Asimismo, Sotomayor (2019) afirma que la feria vocacional aporta información adecuada de las organizaciones de estudios superiores la cual es acogida con interés por los padres y jóvenes que los pone en contacto con las diversas universidades puntualizando el proceso vocacional. En esa línea, Muñoz (2015) explica que las universidades promueven espacios para que los jóvenes entre 16 y 18 años próximos a ingresar a la universidad tengan una fuente de información directa a través de las ferias vocacionales que permite orientar a jóvenes próximos a iniciar la universidad, despertando el interés de estudiantes potenciales que van a la universidad. Por otro lado, Briones y Triviño (2018) puntualiza que la orientación vocacional es permanente para el desarrollo de la persona y define que la feria vocacional es un soporte de apoyo para los jóvenes que terminan la secundaria y siguen el proceso de elegir una carrera profesional o empezar a laborar. Guadagni (2016) también refiere, que la orientación vocacional ayuda al estudiante a prepararse para elegir su profesión y ubicarse en una especialidad especifica de acuerdo a sus habilidades y preferencias y proyectar un futuro con mayor seguridad. Del mismo modo, Sius (2015) refiere que elegir una vocación es trascendente en la persona y se basa en factores internos como preferencias, habilidades, aptitudes para desarrollar su potencial y factores externos como la oferta de profesiones y la exigencia de las evaluaciones. Por otro lado, Retana et al (2018) sostiene que las ferias vocacionales evidencian componentes académicos, emocionales y sociales de la educación científica, humanística y tecnológica escolar que facilita la autonomía y optimiza la autoestima de los estudiantes a través de situaciones lúdicas, la innovación y el emprendimiento estableciendo su vocación.

Según Conley (2014) no es necesario que todos los estudiantes deban tener puntualmente los mismos conocimientos y habilidades para ingresar a la universidad y optar por una carrera. Los intereses que tiene un estudiante para elegir una carrera universitaria ayudan a identificar el conocimiento y las competencias precisas que el estudiante necesita. De acuerdo a Yamen (2019) en la actualidad, las universidades cuentan con bolsas de trabajo para atender los problemas de empleabilidad de los graduados ayudan y atienden los 
problemas de los jóvenes que completan su educación. Por otro lado, Fuller (2020) refiere que, un requisito muy importante para que los jóvenes egresados de la secundaria elijan la universidad es que deben ser motivados en la etapa preprofesional.

En la I.E. Tupac Amaru II SJL, los estudiantes de 5to año carecen de un proyecto de vida y es necesario promover la orientación vocacional con las visitas a las ferias vocacionales programadas por las instituciones superiores para tener una visión amplia de las carreras profesionales y los beneficios que estas brindan. En ese sentido el objetivo de esta investigación fue determinar la relación que existe entre la feria vocacional y elección vocacional en estudiantes de quinto secundaria IE Tupac Amaru II SJL 2020.

\section{MATERIALES Y MÉTODOS}

La investigación fue de tipo aplicada; con un diseño de investigación no experimental, descriptivo, correlacional y de enfoque cuantitativo.

El estudio se realizó entre abril y julio del 2020 contando con una población de 70 estudiantes de 5to año de secundaria de la institución educativa Tupac Amaru II SJL.2020. y la muestra representativa fue de 60 educandos seleccionados de manera probabilística, considerando las particularidades comunes de los estudiantes.

Se empleo el cuestionario con preguntas cerradas para recoger los datos, y se aplicó a los educandos de la IE Tupac Amaru II SJL 2020. El cuestionario de la variable independiente: Feria vocacional, estuvo compuesto por tres dimensiones: beneficios de la feria vocacional, orientación grupal y personalizada y panel profesional con 19 ítems y una escala Likert de "Siempre 5", "Casi siempre 4", “A veces 3", “casi nunca 2", "Nunca 1", por otro lado, la variable dependiente: elección vocacional, estuvo compuesto por tres dimensiones: valor vocacional, socioculturales y elección personal; con 27 ítems; y una escala Likert: "Siempre 5", “Casi siempre 4", “A veces 3", "casi nunca 2", "Nunca 1". La validación de los instrumentos se realizó con el juicio de tres expertos y la prueba de fiabilidad de alfa de Cronbach. Asimismo, se realizó la prueba de normalidad de Kolmogórov - Smirnov.

Se sensibilizó a los maestros, estudiantes y padres de familia, contando con su consentimiento informado; luego, se seleccionó de forma aleatoria a 20 estudiantes para aplicar una prueba piloto de los cuestionarios sobre Orientación vocacional que tuvo una duración de 30 minutos y Elección vocacional que contó un tiempo de 30 minutos las que fueron resueltos por los educandos. 
La información se procesó en el programa Excel en el que se determinó los rangos o niveles de cada una de las variables y dimensiones. También, se utilizó el programa estadístico SPSS v25. Para el análisis descriptivo se realizaron tablas con frecuencias y porcentajes de las variables individuales orientación vocacional y elección vocacional; asimismo, se utilizó las tablas cruzadas por asociación para la comparación de las variables. Por otro lado, el análisis inferencial se realizó con la regresión ordinal simple a través del coeficiente de correlación de Spearman y permitió contrastar y medir el nivel de relación entre la orientación vocacional y la elección vocacional.

La presente investigación consideró conservar la confiabilidad de los resultados, la protección del nombre de los participantes y el respeto. Además, se hace referencia a todos los autores investigados.

\section{RESULTADOS}

En la tabla 1 se presentó la valoración de la feria vocacional donde se pudo observar que el 35\% de estudiantes consideró que la feria vocacional estaba en el nivel regular; $38.3 \%$ opinaron que la feria vocacional se encontraba en el nivel bueno; mientras que el $26.7 \%$ consideraron que la feria vocacional se encontraba en un nivel excelente. Observándose la predominancia de nivel bueno para la feria vocacional.

\section{Tabla 1.}

Feria vocacional en el nivel logrado

\begin{tabular}{llcc}
\hline \multicolumn{1}{c}{ Niveles } & Frecuencia & Porcentaje \\
\hline \multirow{4}{*}{ Válido } & Regular & 21 & 35.0 \\
& Bueno & 23 & 38.3 \\
& Excelente & 16 & 2.7 \\
& Total & 60 & 100.0 \\
\hline
\end{tabular}

Fuente: Cuestionario de feria vocacional realizado el día 25 de junio del 2020

En la tabla 2 se presentó la valoración de la elección vocacional donde se pudo observar que el $33.3 \%$ de estudiantes manifestó que la elección vocacional se encontraba en el nivel regular; el 35\% de estudiantes opinaron que la elección vocacional era de nivel bueno; y el 31.7\% de estudiantes refirieron que la elección vocacional estaba en un nivel excelente. Observándose la predominancia de nivel Bueno para la elección vocacional. 
Tabla 2.

Nivel de predominancia Elección vocacional

\begin{tabular}{clcc}
\hline & \multicolumn{1}{c}{ Niveles } & Frecuencia & Porcentaje \\
\hline \multirow{3}{*}{ Válido } & Regular & 20 & 33,3 \\
& Bueno & 21 & 35,0 \\
& Excelente & 19 & 31,7 \\
& Total & 60 & 100,0 \\
\hline
\end{tabular}

Fuente: Cuestionario de Elección vocacional realizado el día 25 de junio del 2020

En la tabla 3 se presenta los niveles de comparación entre la feria vocacional y la elección vocacional observando que el $6.7 \%$ de estudiantes consideraron que la feria vocacional y la elección vocacional estaban en un nivel excelente; mientras que el 10\% de estudiantes consideró que el nivel era bueno.

\section{Tabla 3.}

Tabla cruzada de las variables Feria vocacional y Elección vocacional.

\begin{tabular}{|c|c|c|c|c|c|c|}
\hline \multicolumn{3}{|l|}{ Variables } & \multicolumn{3}{|c|}{ Elección Vocacional } & \multirow[b]{2}{*}{ Total } \\
\hline & Niveles & & Regular & Bueno & Excelente & \\
\hline \multirow{6}{*}{$\begin{array}{l}\text { Feria } \\
\text { vocacional }\end{array}$} & \multirow{2}{*}{ Regular } & Recuento & 7 & 8 & 6 & 21 \\
\hline & & $\%$ del total & $11.7 \%$ & $13.3 \%$ & $10.0 \%$ & $35.0 \%$ \\
\hline & \multirow{2}{*}{ Bueno } & Recuento & 8 & 6 & 9 & 23 \\
\hline & & $\%$ del total & $13.3 \%$ & $10.0 \%$ & $15.0 \%$ & $38.3 \%$ \\
\hline & \multirow{2}{*}{ Excelente } & Recuento & 5 & 7 & 4 & 16 \\
\hline & & $\%$ del total & $8.3 \%$ & $11.7 \%$ & $6.7 \%$ & $26.7 \%$ \\
\hline \multirow[t]{2}{*}{ Total } & & Recuento & 20 & 21 & 19 & 60 \\
\hline & & $\%$ del total & $33.3 \%$ & $35.0 \%$ & $31.7 \%$ & $100.0 \%$ \\
\hline
\end{tabular}

La tabla 4 muestra que los resultados de la relación entre las variables feria vocacional y elección vocacional fue de una correlación de $r=-0.00$ con una sigma de $p=0.999$ mayor a 0.05 lo que indicó que la relación fue negativa y escasa entre las variables de investigación, por lo que se aceptó la hipótesis nula y se rechazó la hipótesis alterna. 


\section{Tabla 4.}

Correlación de Spearman entre Feria vocacional y Elección vocacional

\begin{tabular}{|c|c|c|c|c|}
\hline & Variables & & $\begin{array}{c}\text { Feria } \\
\text { vocacional }\end{array}$ & $\begin{array}{c}\text { Elección } \\
\text { vocacional }\end{array}$ \\
\hline \multirow{6}{*}{$\begin{array}{l}\text { Rho de } \\
\text { Spearman }\end{array}$} & \multirow{3}{*}{$\begin{array}{l}\text { Feria } \\
\text { vocacional }\end{array}$} & Coeficiente de correlación & 1.000 & .000 \\
\hline & & Sig. (bilateral) & & .999 \\
\hline & & $\mathrm{N}$ & 60 & 60 \\
\hline & \multirow{3}{*}{$\begin{array}{l}\text { Elección } \\
\text { vocacional }\end{array}$} & Coeficiente de correlación & .000 & 1.000 \\
\hline & & Sig. (bilateral) & .999 & . \\
\hline & & $\mathrm{N}$ & 60 & 60 \\
\hline
\end{tabular}

\section{DISCUSIÓN}

Los hallazgos muestran que la relación entre feria vocacional y elección vocacional tienen una correlación con $\mathrm{r}=0,000$; y un grado sigma de $\mathrm{p}=0,999 ;>0,05$, que determinan que no existe evidencia suficiente que relacione significativamente a las variables de estudio. Sin embargo, Olivares y Quezada (2013) en un estudio en relación a la posibilidad de considerar la conveniencia de continuar con las ferias vocacionales, encontró que el $91 \%$ de participantes estuvo a favor de seguir realizando estos eventos y el impacto de las ferias vocacionales en la orientación vocacional era parcial. Asimismo, Panina et al (2020) en un estudio sobre la carrera vocacional realizada antes y durante la cuarentena de la COVID 19 refirieron que es necesario mejorar el componente psicológico de la formación de los especialistas a través de consultas psicológicas y pedagógicas individuales, ampliar y demostrar las posibilidades del mercado laboral y el empleo temporal, organizar webinars sobre preparación de currículums y autopresentación para reuniones con empleadores

Por otro lado, la relación entre los beneficios de la feria vocacional y la elección vocacional muestran una correlación $\mathrm{r}=0.032$ y una sigma de $\mathrm{P}=0.808>$ a 0.05 determinando que no existe un nivel de relación significante entre las variables de estudio. En tal sentido, Loc et al (2020) concluyó que los estudiantes son conscientes de la importancia de la orientación vocacional y los factores que afectan su elección de carrera. Por lo que, los propios estudiantes, sus familias y la escuela deben tener nuevos enfoques en la orientación vocacional para los estudiantes. Sugiriendo que el colegio, los maestros y las familias deben escuchar los deseos de sus hijos, los estudiantes deben ser conscientes 
de sus propias condiciones, economía doméstica, intereses y capacidad académica y en la escuela, se debe prestar mucha atención a la orientación vocacional para los estudiantes, organizar actividades extracurriculares para que los estudiantes puedan experimentar y aprender muchas profesiones. Sin embargo, refieren Olivares y Quezada (2013) los beneficios de la feria vocacional tienen que ver con la orientación e información que reciben los estudiantes para conocer los beneficios que brinda la educación superior.

En cuanto a la relación entre la elección vocacional y el valor vocacional los resultados muestran una relación inversa escasa con una correlación de -0.073 y un grado sigma de p=0.80;> a 0.05 que establece que no existe una relación significante entre las variables. Estos hallazgos son similares a los que encontró, Bulgarelli et al (2017) en su investigación que, en cuanto a las tareas vocacionales, la parte académica cumplió con el logro esperado motivado por una orientación adecuada, una alta socialización y acompañamiento especial; sin embargo, en el aspecto de los factores vocacionales existió dispersidad entre los estudiantes participantes y que la valoración de los factores vocacionales en la elección de una profesión no necesariamente aseguran ni predicen la condición académica de logro. Por otro lado, un estudio de Cedefop (2014) concluyo que los gobiernos deben promover formas superiores de educación vocacional, comprometiéndose con la industria y grupos profesionales para promover el prestigio de los programas y resultados, y también las escuelas y los maestros de escuela que juegan un papel importante en la reparación de la paridad de estima. problemas dentro de la educación y proponer la formación profesional como una opción educativa viable, basada en los resultados de empleabilidad.

Del mismo modo, la relación entre la elección vocacional y la elección personal muestran una correlación con 0.001 y un grado sigma de $\mathrm{p}=0.096>$ a 0.05 ; que determina que no existe una relación significativa entre las variables. Aunque, Barrera (2015) indica que la elección personal implica tomar una decisión en la que previamente se ha reflexionado y para que esa elección sea adecuada el estudiante debe tener una formación y orientación durante sus estudios de secundaria.; que le permitan elegir sus metas y determinen su futuro profesional. Esto significa optar por un modo de vida, por esa razón es una de las decisiones más importantes, porque, sino elige la carrera adecuada terminará con una insatisfacción laboral. En ese sentido Guadamuz y Rodríguez (2020) en un estudio concluyó que ofrecer experiencias y divulgar la información sobre la oferta académica de 
una manera interactiva y vivencial contribuye al aprendizaje significativo de las personas visitantes, ya que adquieren un papel activo, se despierta la curiosidad y una oportunidad de diálogo más fluido entre las personas informantes y visitantes. Asimismo, hacerlo de esta manera puede incluso cambiar la percepción de las personas visitantes sobre la carrera y abre la posibilidad de erradicar mitos sobre ella.

\section{CONCLUSIONES}

Se cumplió el objetivo propuesto encontrando que no existe una relación significativa que relacione las variables feria vocacional y la elección vocacional. Por qué no existe una planificación y orientación con personas capacitadas para apoyar la elección de carreras profesionales a los estudiantes. Por otro lado, los estudiantes no indagan sobre la profesión que les ofrece su entorno y tampoco desarrollan otras actividades después de las horas de clase que no les facilita que estudiar después de terminar la secundaria y las organizaciones públicas ni privadas les ofrecen los suficientes espacios para su orientación vocacional.

Asimismo, la relación de la feria vocacional con las dimensiones elección vocacional y la elección personal muestran una relación escasa y poco relevante y significativa lo que implica que en las escuelas se tiene que implementar una estrategia que promueva la interacción entre docente y alumno encaminada en un modelo de formación profesional que involucre el aspecto vocacional teniendo en cuenta las características de la edad, las potenciales competencias y habilidades, la diversidad de intereses vinculados con una motivación autónoma y controlada en el proceso de orientación de la orientación vocacional hasta la elección personal de una carrera que sea de su agrado y asegure el futuro de la persona.

\section{REFERENCIAS BIBLIGRÁFICAS}

Barrera, M (2015). Orientación vocacional para una acertada elección de carreras e la educación superior. Investigación arbitrada.2 (3) p. 25 - 34. Recuperado de https://bit.ly/3oqN9tk

Briones, Y., Triviño, J. (2018). Factores que intervienen para elegir carreras universitarias. Enfermería global. 1(37) p.200 -207. Recuperado de https://bit.ly/31lubvr 
Bulgarelli, R., Rivera, J., Fallas, M. (2017) El proceso vocacional del estudiantado universitario en condición de logro y rezago académico: Un análisis desde el enfoque evolutivo de Donald Super. Revista Electrónica Educare, 1(21). Recuperado de https://bit.ly/32QOvVG

Cedefop. (2014) Attractiveness of Initial Vocational Education and Training: Identifying What Matter. Luxembourg: Office of the European Union. Recuperado de https://bit.ly/3pw7ZGX

Conley, D. (2014). New conceptions of college and career ready: A profile approach to admission. Journal of College Admission, 223, 12-23. Recuperado de https://bit.ly/3rywANW

Expo - Estudiante (2019-2)-Catalogue-A4 ¡Estudia en el extranjero! Recuperado de: https://bit.ly/3opkswQ

Fuller, E. (2020). The most stressful thing in my life: when it's time to confront life beyond college. Leadership, 49(5), pág. 18-22. Recuperado de https://leadership.acsa.org/the-most-stressful-thing-in-my-life

Guadagni (2016). Ingreso a la Universidad en Ecuador, Cuba y argentina. CEA, 5(44), 1-14. Recuperado de https://www.rlcu.org.ar/recursos/E_0000046_004_cea_numero_44.pdf

Guadamuz, J. y Rodríguez, D. (2020). Divulgación de la carrera de Bibliotecología: Una mirada desde la Feria Vocacional de la Universidad de Costa Rica (UCR), 2019. e-Ciencias de la Información, 10(2).

Doi: http://dx.doi.org/10.15517/eci.v10i2.39366

Loc, N., Tong, D., Nguyen Tram, N., Ky, V., Nhut, H., y Hai, N. (2020). Students' opinions on vocational guidance: a case study. European Journal of Education Studies, 6(10). https://doi.org/10.5281/zenodo.3604498

Muñoz, Z. (2015). Las ferias como herramienta de comunicación y promoción del sector educativo, una aproximación al marketing ferial universitario en España, el caso de Aula, Talent y Unitour Valencia 2012 (Tesis doctoral). Recuperado de https://bit.ly/3DqfMeb

Olivares, M., y Quezada, J. (2013). Impact of the vocational fairs in the vocational orientation. Recuperado de https://bit.ly/3ontni6 
Panina, S., Arkhipova, S., Parnikova, T., Sergina, E., y Sleptsova, M. (2020). Student Career Choices before and during Quarantine Measures. Propósitos y Representaciones, 8 (3), pág.711.

Doi: http://dx.doi.org/10.20511/pyr2020.v8nSPE3.711

Pronabec (2019). Memoria anual. Recuperado https://bit.ly/31wCC6A

Retana, D., Vázquez, B., y Camacho, M. (2018). Las Ferias de Ciencia y Tecnología de Costa Rica y sus aportes a la educación secundaria. Actualidades Investigativas en Educación, 18(2), 309-352.Doi: https://dx.doi.org/10.15517/aie.v18i2.33170

Sánchez, M. (2017). Marco conceptual de la orientación para el desarrollo profesional y personal. En Sánchez García, M.F. (coord.), Orientación profesional y personal (pp. 15-39). Madrid, España: UNED.

Sius, M. (2015) GPS Vocacional psicóloga infanto-juvenil. Recuperado de https://bit.ly/330zwZw

Thenmozhi, C. (2018). Vocational Guidance and Its Strategies. Shanlax International Journal of Education. 7 (1), pág. 20-23. Doi: 10.5281/zenodo. 2545060

Universidad de Costa Rica. Oficina de Orientación. (2019). Feria vocacional UCR. Recuperado de http://orientacion.ucr.ac.cr/feriavocacional/

Yamen, V. (2019). Development features of the students' career building skills. International Journal of Humanities and Natural Sciences, 7(1), 18-21.

Doi: http://dx.doi.org/10.20511/pyr2020.v8nSPE3.711 